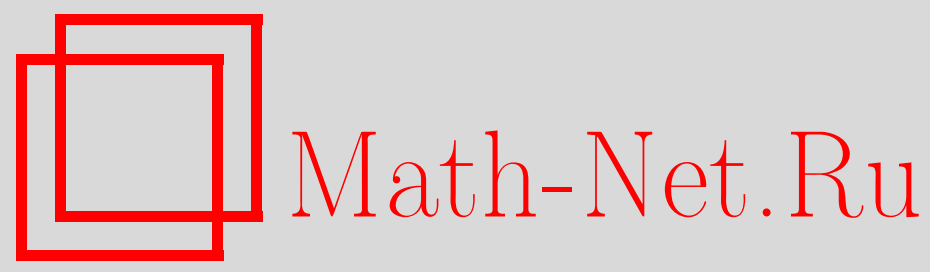

В. Н. Карпушкин, Число компонент дополнения к гиперповерхности уровня частично гармонического полинома, $\mathrm{Ma-}$ тем. заметки, 1997, том 62, выпуск 6, 831-835

DOI: https://doi.org/10.4213/mzm1672

Использование Общероссийского математического портала Math-Net.Ru подразумевает, что вы прочитали и согласны с пользовательским соглашением http://www . mathnet.ru/rus/agreement

Параметры загрузки:

IP : 54.162 .27 .143

26 апреля 2023 г., 14:45:20

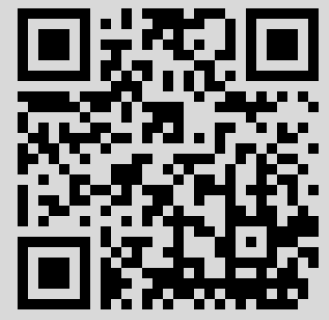




\section{ЧИСЛО КОМПОНЕНТ ДОПОЛНЕНИЯ К ГИПЕРПОВЕРХНОСТИ УРОВНЯ ЧАСТИЧНО ГАРМОНИЧЕСКОГО ПОЛИНОМА}

\section{В.Н. Карпушкин}

Известно, что число компонент дополнения к множеству нулей полинома степени $m$ в $\mathbb{R}^{n}$ не превосходит $m^{n}+O\left(m^{n-1}\right)$. В работе рассматриваются $k$-гармонические полиномы $F$ в $\mathbb{R}^{n}$, т.е. удовлетворяющие уравнению Лапласа по части переменных: $\left(\partial^{2} / \partial x_{1}^{2}+\cdots+\partial^{2} / \partial x_{k}^{2}\right) F=0$. Здесь $1 \leqslant k \leqslant n, n \geqslant 2$. Показано, что число компонент дополнения к гиперповерхности уровня такого полинома степени $m$ не превосходит $2 m^{n-1}+O\left(m^{n-2}\right)$. Получены более точные оценки при предположении компактности множества особых точек гиперповерхности уровня или неособости главной однородной части $k$-гармонического полинома.

Библиографияя: 8 названий.

Известно, что число компонент дополнения к множеству нулей полинома степени $m$ в $\mathbb{R}^{n}$ не превосходит $m^{n}+O\left(m^{n-1}\right)$ (см. [1]). В настоящей работе рассматриваются $k$-гармонические полиномы $F$ в $\mathbb{R}^{n}$, т.е. удовлетворяющие уравнению Лапласа по части переменных:

$$
\left(\frac{\partial^{2}}{\partial x_{1}^{2}}+\cdots+\frac{\partial^{2}}{\partial x_{k}^{2}}\right) F=0, \quad 1 \leqslant k \leqslant n, \quad n \geqslant 2 .
$$

Показано, что число компонент дополнения к гиперповерхности уровня полинома $F$ не превосходит $2 m^{n-1}+O\left(m^{n-2}\right)$. Получены более точные оценки при предположении компактности множества особых точек гиперповерхности уровня или неособости главной однородной части $k$-гармонического полинома.

1. Формулировка результатов. Пусть $W_{c}$-гиперповерхность уровня $c$ для $k$-гармонического полинома $F$ степени $m$ в $\mathbb{R}^{n}$. Пусть $V$ - регулярное пересечение квадрики и гиперповерхности степени $m$ в $\mathbb{C P}^{n}$. Сумма чисел Бетти для $V$ с коэффициентами в $\mathbb{Z}_{2}$, т.е. $\operatorname{rank} H_{*}(V)$, зависит только от $m, n$ и равна

$$
\begin{array}{ll}
n-2+2 m+2 \frac{(m-1)^{3}(m-1)^{n-2}-1}{m(m-2)}, & \text { если } n \text { четное, } m \geqslant 3 ; \\
n-5+2 m+2\left(\frac{(m-1)^{3}(m-1)^{n-2}-1}{m(m-2)}+\frac{1}{m}\right), & \text { если } n \text { нечетное, } m \geqslant 3
\end{array}
$$

(см. $[1$, c. 58]).

Работа выполнена при финансовой поддержке фонда INTAS, грант № 4773. 
ТЕорема 1. Выполнено неравенство

$$
\operatorname{rank} H_{0}\left(\mathbb{R}^{n} \backslash W_{c}\right) \leqslant \operatorname{rank} H_{*}(V) .
$$

ТЕорема 2. Предположим, что множество особых точек гиперповерхности $W_{c}$ компактно. Тогда

$$
\operatorname{rank} H_{0}\left(\mathbb{R}^{n} \backslash W_{c}\right) \leqslant \frac{1}{2} \operatorname{rank} H_{*}(V)+1 .
$$

ОПРЕДЕЛЕНИЕ. Числом Петровского назовем число целых точек строго внутри куба $(0, m)^{n}$, лежаших на проходящей через центр куба гиперплоскости, перпендикулярной главной диагонали.

Обозначим его через

$$
\Pi_{n}(m)=\#\left\{k=\left(k_{1}, \ldots, k_{n}\right): 0<k_{s}<m, \sum k_{s}=\frac{1}{2} m n\right\}
$$

(см. [2]).

Для неособой гиперповерхности $U$ степени $m$ в $\mathbb{C P}^{n-1}$ сумма чисел Бетти по $\mathbb{Z}_{2}$, т.е. $\operatorname{rank} H_{*}(U)$, зависит только от $m, n$ и равна

$$
\frac{(m-1)^{n}+(-1)^{n}}{m}+n+(-1)^{n+1}
$$

(см. $[1$, с. 52$])$.

ТЕорема 3. Предположим, что главная однородная часть полинома $F$ неособая в $\mathbb{R}^{n}$. Тогда

$$
\operatorname{rank} H_{0}\left(\mathbb{R}^{n} \backslash W_{c}\right) \leqslant \begin{cases}\frac{1}{2}\left(\operatorname{rank} H_{*}(U)+\Pi_{n}(m)+1\right)+1, & \text { еслип четное, } \\ \operatorname{rank} H_{*}(U)+1, & \text { еслип нечетное. }\end{cases}
$$

Tеорема 4. Пусть $W_{c}$ содержит конечное множество критических точек полинома $F$. Тогда $\operatorname{rank} H_{0}\left(\mathbb{R}^{n} \backslash W_{c}\right) \geqslant \operatorname{rank} H_{0}\left(W_{c}\right)+1$.

СлЕДСтвиЕ. Пусть гиперповерхность $W_{c}$ содержит конечное число критических точек полинома $F$. Тогда

1) $\operatorname{rank} H_{0}\left(W_{c}\right) \leqslant \frac{1}{2} \operatorname{rank} H_{*}(V)$;

2) если предположить, что главная однородная часть полинома $F$ неособая, mo

$$
\operatorname{rank} H_{0}\left(W_{c}\right) \leqslant \begin{cases}\frac{1}{2}\left(\operatorname{rank} H_{*}(U)+\Pi_{n}(m)+1\right) & \text { при п четном, } \\ \operatorname{rank} H_{*}(U) & \text { при п нечетном. }\end{cases}
$$


ЗАмЕчАнИЕ 1. В настоящей работе используются теоремы алгебраической геометрии и принцип максимума. Если предположить, что $k=n$, то можно воспользоваться теоремой Куранта (см. [3]). Пусть сужение на сферу с центром в $0 \in \mathbb{R}^{n}$ главной однородной части гармонического полинома $F$ имеет на гиперповерхности уровня 0 только невырожденные критические точки. Тогда

$$
\operatorname{rank} H_{0}\left(\mathbb{R}^{n} \backslash W_{c}\right) \leqslant 2\left(C_{n+m-3}^{n-1}+1\right), \quad n \geqslant 2
$$

(cм. [4]).

ЗАМЕЧАНИЕ 2. Если

$$
F=\sum_{j} p_{j}\left(x_{1}, \ldots, x_{k}\right) f_{j}\left(x_{k+1}, \ldots, x_{n}\right),
$$

где $p_{j}$ - полиномы от $x_{1}, \ldots, x_{k}$, то $F$ будет $k$-гармоничен при условии, что все $p_{j}-$ гармонические полиномы.

ЗАмЕчаниЕ 3 . Пусть главная однородная часть полинома $F$ неособая в 0. Тогда существует компакт в $\mathbb{R}^{n}$, вне которого $\operatorname{grad} F$ не обращается в 0 . Таким образом, каждая гиперповерхность $W_{c}$ уровня $c$ имеет компактное множество особых точек.

2. Доказательство результатов. Докажем сперва теорему 1 . Пусть $S_{r}^{n-1}-$ сфера радиуса $r$ в $\mathbb{R}^{n}$ с центром в 0.

Лемма 1. Пусть $W_{c}$ - гиперповерхность уровня $с$ для $k$-гармонического полинома $F$ в $\mathbb{R}^{n}(n \geqslant 2)$. Тогда найдется $r_{0}$ такое, что

$$
\operatorname{rank} H_{0}\left(S_{r}^{n-1} \backslash W_{c}\right) \geqslant \operatorname{rank} H_{0}\left(\mathbb{R}^{n} \backslash W_{c}\right)
$$

npu $r \geqslant r_{0}$.

ДокАЗАТЕЛьство. Пусть $A$ - ограниченная компонента множества $\mathbb{R}^{n} \backslash W_{c}$. В силу того что $F$ - полином, размерность $A$ равна $n$. Тогда $A$ - открытое множество и строго внутри $A$ лежит экстремум полинома $F$. В силу $k$-гармоничности полинома $F$ получим противоречие. Лемма 1 доказана.

Лемма 2. Для всех $c \in \mathbb{R}, r>0$ найдется $\varepsilon$ такое, что $W_{c \pm \varepsilon} \cap S_{r}^{n-1}$ - неособые многообразия или пустые множества и

$$
\operatorname{rank} H_{0}\left(W_{c+\varepsilon} \cap S_{r}^{n-1}\right)+\operatorname{rank} H_{0}\left(W_{c-\varepsilon} \cap S_{r}^{n-1}\right) \geqslant \operatorname{rank} H_{0}\left(S_{r}^{n-1} \backslash W_{c}\right) .
$$

ДоКАЗАТЕЛЬСТВО следует из конечности числа критических значений у сужения полинома $F$ на сферу $S_{r}^{n-1}, r>0$. Последнее вытекает из следствия 2.8 работы [5].

Таким образом, из теоремы Смита $[1$, с. 53$]$ получаем, что

$$
\operatorname{rank} H_{0}\left(S_{r}^{n-1} \backslash W_{c}\right) \leqslant \operatorname{rank} H_{*}(V) .
$$

Отсюда и из леммы 1 следует теорема 1. 
ДОКАЗАТЕЛЬСТВО ТЕОРЕМЫ 2. Из следствия 2.8 работы [5] получим, что для всякого $c$ существует $r_{0}$ такое, что при $r \geqslant r_{0} W_{c} \backslash S_{r}^{n-1}$ есть регулярное пересечение. Теперь из теоремы Смита [1] и леммы 1 получим доказательство теоремы 2.

ДоКАЗАТЕЛЬСТВО ТЕОРЕМЫ 3. Пусть $F=f_{0}+\cdots+f_{m}, f_{i}$ - однородная форма степени $i$ полинома $F$. Положим

$$
D=\left\{x \in \mathbb{R P}^{n-1}: f_{m}(x)=0\right\}, \quad B_{r}=\left\{x \in S_{r}^{n-1}: f_{m}(x)=0\right\}
$$

Легко видеть, что $\operatorname{rank} H_{0}\left(B_{r}\right) \leqslant 2 \operatorname{rank} H_{0}(D)$ при всех $r>0$. Из неособости $D$, работ [2], [6] и теоремы Смита [1] при четном $n$ имеем

$$
\operatorname{rank} H_{0}(D) \leqslant \frac{1}{4}\left(\Pi_{n}(m)+1+\operatorname{rank} H_{*}(U)\right)
$$

при нечетном $n$ из теоремы Смита [1] следует, что

$$
\operatorname{rank} H_{0}(D) \leqslant \frac{1}{2} \operatorname{rank} H_{*}(U)
$$

Таким образом, получили оценку сверху $\operatorname{rank} H_{0}\left(B_{r}\right)$.

Из неособости формы $f_{m}$ следует, что $B_{r}-$ неособое многообразие при всех $r>0$. Для каждого значения $c$ найдется $r_{0}(c)$ такое, что при $r \geqslant r_{0}(c) W_{c} \cap S_{r}^{n-1}$ будет малым возмущением для $B_{r}$, так что топология $W_{c} \cap S_{r}^{n-1}$ такая же, как у $B_{r}$, и, в частности, $W_{c} \cap S_{r}^{n-1}$ - неособое многообразие. Отсюда и из леммы 1 следует теорема 3.

ДОКАЗАТЕЛЬСТВО ТЕОРЕМЫ 4 . Из принципа максимума для $k$-гармонических полиномов и конечности множества критических точек полинома $F$ на $W_{c}$ следует, что $W_{c}$ не имеет компонент связности, состоящих из особых точек. Таким образом, каждая компонента связности множества $W_{c}$ содержит неособые точки и поэтому (см. [7]) имеет хотя бы один $(n-1)$-мерньй цикл в гомологиях с коэффициентами в $\mathbb{Z}_{2}$. Ранг группы $(n-1)$-гомологий $W_{c}$ с коэффициентами в $\mathbb{Z}_{2}$ будет, таким образом, не меньше, чем число компонент связности $W_{c}$. Однако, группа 0 -мерных гомологий дополнений к гиперповерхности, приведенная по модулю точки, двойственна по Александеру к группе $H_{n-1}\left(W_{c}, \mathbb{Z}_{2}\right)$. Отсюда имеем $\operatorname{rank} H_{0}\left(\mathbb{R}^{n} \backslash W_{c}\right) \geqslant \operatorname{rank} H_{0}\left(W_{c}\right)+1$. Теорема 4 доказана.

ЗАмечаниЕ 4. Доказанные вьше теоремы уточняют соответствуюшие результаты работы [8] и исправляют ошибки в приведенных там доказательствах.

ЗАмЕчаниЕ 5. Для получения $k$-гармонического $(k=2 p)$ полинома $F$ с неособой главной однородной частью достаточно взять

$$
F=f\left(x_{1}, \ldots, x_{k}\right)+g\left(x_{k+1}, \ldots, x_{n}\right)+h\left(x_{1}, \ldots, x_{n}\right),
$$

где $f, g$ - однородные полиномы степени $m, f=\operatorname{Re} Q$, где $Q$ - однородньй полином в $\mathbb{C}^{p}$ с изолированной критической точкой в $0 \in \mathbb{C}^{p}, g$ имеет изолированную критическую точку в $0 \in \mathbb{R}^{n-k}$. Здесь $h k$-гармоничен и имеет степень меньше, чем $m$.

Автору неизвестно, точны ли по порядку (по степени) полученные в работе оценки. 


\section{СПИСОК ЦИТИРОВАННОЙ ЛИТЕРАТУРЫ}

[1] Гудков Д.А. Топология вещественных проективных алгебраических многообразий // УМН. 1974. Т. 29. №4. С. 3-79.

[2] Арнольд В. И. Индекс особой точки векторного поля, неравенства Петровского-Олейник и смешанные структуры Ходжа // Функцион. анализ и его прилож. 1978. Т. 12. №1. С. 1-14.

[3] Курант Р., Гильберт Д. Методы математической физики. Т. 1. М.: ИЛ, 1933.

[4] Карпушкин В.Н.Число компонент дополнения к гиперповерхности уровня гармонического полинома // Вестн. МГУ. Сер. 1. Матем., мех. 1981. №4. С. 3-4.

[5] Милнор Дж. Особые точки комплексных гиперповерхностей. М.: Мир, 1971.

[6] Петровский И.Г., Олейник О.А. О топологии действительных алгебраических гиперповерхностей // Изв. АН СССР. Сер. матем. 1949. Т. 13. С. 389-402.

[7] Borel A., Haefliger A. La classe d'homologie fondamentale des variétés analytiques // Ann. Inst. Fourier (Grenoble). 1958. V. 2. P. 49-96.

[8] Карпушкин В. Н. Точные по порядку оценки числа компонент дополнения к нулям гармонических полиномов // Функцион. анализ и его прилож. 1985. Т. 19. № 4. С. 55-60.

Институт проблем передачи информации РАН

Поступило 22.09 .95

Исправленный вариант

15.05.97 